\title{
Effect of acupuncture on women with poor ovarian response: a study protocol for a multicenter randomized controlled trial
}

\author{
Huanfang $\mathrm{Xu}^{1 \dagger}$, Chensi Zheng ${ }^{1 \dagger}$, Liyun $\mathrm{He}^{2}$, Tongsheng $\mathrm{Su}^{3}$, Huidan Wang ${ }^{4}, \mathrm{Yu} \mathrm{Li}{ }^{5}$, Cui Zhao ${ }^{6}$, Cuilian Zhang ${ }^{7}$, \\ Yang Bai ${ }^{8}$, Guoqing Tong ${ }^{9}$, Li Chen ${ }^{10}$, Fang Zhao ${ }^{11}$, Huisheng Yang ${ }^{1}$, Mingzhao Hao ${ }^{1}$, Yaqian Yin ${ }^{1}$, Li Yang ${ }^{1}$, \\ Yigong Fang ${ }^{1^{*}}$ and Baoyan Liu ${ }^{2^{*}}$
}

\begin{abstract}
Background: Poor ovarian response (POR), a manifestation of low ovarian reserve and ovarian aging, leads to a significant reduction in the pregnancy rate after in vitro fertilization-embryo transfer. Acupuncture has increasingly been used to improve the ovarian reserve. The purpose of this study will be to evaluate the effect of acupuncture on increasing the number of retrieved oocytes after controlled ovarian hyperstimulation in women with POR.

Methods: This will be a multicenter randomized controlled trial. A total of 140 women with POR will be randomly assigned to receive acupuncture or nontreatment for 12 weeks before controlled ovarian hyperstimulation. The primary outcome will be the number of retrieved oocytes. The secondary outcomes will be antral follicle counts, serum levels of anti-Müllerian hormone, basal serum levels of follicle stimulating hormone, luteinizing hormone and estradiol levels, scores from the self-rating anxiety scale, fertilization rates, cleavage rates, available embryo rates, and high-quality embryo rates. The safety of acupuncture will also be assessed.
\end{abstract}

Discussion: The results of this trial will help to determine the effectiveness of acupuncture in the treatment of POR. This may provide a new treatment option for patients with POR and their physicians.

Trial registration: AMCTR-IPR-18000198. Registered on 10 August 2018.

Keywords: Acupuncture, Poor ovarian response, Number of retrieved oocytes, In vitro fertilization-embryo transfer, Randomized controlled trial

\section{Background}

Poor ovarian response (POR) indicates a reduction in follicular response to the controlled ovarian hyperstimulation $(\mathrm{COH})$ during in vitro fertilization (IVF) cycles, and it results in a reduced number of retrieved oocytes [1]. It is believed that approximately $9-24 \%$ of women

\footnotetext{
*Correspondence: fangyigong@163.com; liuby5505@139.com

†Huanfang Xu and Chensi Zheng are co-first authors.

'Institute of Acupuncture and Moxibustion, China Academy of Chinese Medical Sciences, Beijing, China

${ }^{2}$ Institute of Basic Research in Clinical Medicine, China Academy of Chinese Medical Sciences, Beijing, China

Full list of author information is available at the end of the article
}

who undergo IVF show POR during $\mathrm{COH}$ [2]. Women with POR usually experience less follicular development in $\mathrm{COH}$ cycles, low blood estrogen levels, high doses of gonadotrophin, high cycle cancelation rates, decreased numbers of retrieved follicles, and low rates of clinical pregnancy [3]. Therefore, POR often leads to an unsatisfactory outcome in assisted reproductive technology (ART), which seriously affects physical and mental health as well as quality of life.

An individualized $\mathrm{COH}$ protocol designed according to the assessment of ovarian reserve is the key to success of POR treatment in the first and multiple cycles.

(c) The Author(s). 2020 Open Access This article is licensed under a Creative Commons Attribution 4.0 International License, which permits use, sharing, adaptation, distribution and reproduction in any medium or format, as long as you give appropriate credit to the original author(s) and the source, provide a link to the Creative Commons licence, and indicate if changes were made. The images or other third party material in this article are included in the article's Creative Commons licence, unless indicated otherwise in a credit line to the material. If material is not included in the article's Creative Commons licence and your intended use is not permitted by statutory regulation or exceeds the permitted use, you will need to obtain permission directly from the copyright holder. To view a copy of this licence, visit http://creativecommons.org/licenses/by/4.0/ The Creative Commons Public Domain Dedication waiver (http://creativecommons.org/publicdomain/zero/1.0/) applies to the data made available in this article, unless otherwise stated in a credit line to the data. 
Various protocols of ovarian stimulation, including gonadotrophin-releasing hormone $(\mathrm{GnRH})$ agonist protocols [4], GnRH antagonist protocols [5], and some nontraditional protocols (microstimulation, natural cycles, luteal ovulation, etc.), have been proposed for optimizing ART outcomes; however, challenges remain for women with poor responses to $\mathrm{COH}$. Many pretreatment modalities (including growth hormone, estrogen, dehydroepiandrosterone, and oral contraceptives) have been used prior to ovulation induction to increase the success rate for women undergoing ART [6-8], and yet evidence for the effectiveness of these pretreatments are limited [9].

Acupuncture, an important part of traditional Chinese medicine, has gained worldwide popularity for improving reproductive outcomes in women undergoing ART. Although high-quality research is still needed, several lines of evidence have shown that acupuncture is effective at increasing the number of retrieved oocytes [10], improving clinical pregnancy rate and live birth rate [11, 12], and relieving anxiety [13] in women who undergo in vitro fertilization-embryo transfer (IVF-ET). Low ovarian reserve often indicates a poor ovarian response in $\mathrm{COH}$. Although acupuncture has not been studied for POR, its effectiveness in improving ovarian reserve for women with diminished ovarian reserve (DOR) or premature ovarian insufficiency (POI) has been reported. The results of our previous studies showed that acupuncture significantly lowered serum levels of folliclestimulating hormone (FSH) and luteinizing hormone (LH), raised serum levels of anti-Müllerian hormone $(\mathrm{AMH})$ and estradiol (E2), and increased antral follicle count (AFC) in patients with DOR [14, 15] or POI [16]. Acupuncture was found to regulate the menstrual cycle and to improve perimenopausal symptoms $[14,15]$.

Given its effect on the improvement of ovarian reserve and ART outcomes, acupuncture is expected to be a new choice for the treatment of POR. Furthermore, POR patients treated by transcutaneous electrical acupoint stimulation (TEAS), a noninvasive acupuncture-like therapy, showed significant improvement on basal serum sex hormones, $\mathrm{AMH}$, the number of retrieved oocytes, and fertilization rate $[17,18]$, which indirectly indicated the effect of acupuncture for POR. Therefore, we aim to conduct a multicenter, randomized controlled trial (RCT) to evaluate the effectiveness of acupuncture on women with POR.

\section{Methods}

\section{Study design}

This will be an exploratory multicenter randomized controlled trial comparing acupuncture with nontreatment. A total of 140 women with POR will be recruited from the following 9 hospitals in China: Shanxi Provincial Hospital of Chinese Medicine, Shandong University
Reproductive Hospital, Sun Yat-sen Memorial Hospital of the Sun Yat-sen University, Lanzhou University First Hospital, People's Hospital of Henan Province, Huazhong University of Science and Technology Reproductive Medicine Center of Tongji Medical College, Shanghai Shuguang Hospital, Nanjing General Hospital of Nanjing Military Command, and Luoyang Women and Children Health Care Center. This protocol is in accordance with the principles of the Declaration of Helsinki. It was approved by the ethics committee of each participating hospital. Written informed consent will be obtained from patients prior to enrolment.

Eligible patients will be randomly assigned to receive acupuncture or nontreatment for 12 weeks, which will be followed by an IVF cycle. Outcomes will be assessed at baseline, after acupuncture or nontreatment intervention, and after the IVF cycle. The flowchart and study design schedule are presented in Fig. 1 and Table 1, respectively. This protocol has been registered at Acupuncture-Moxibustion Clinical Trial Registry (AMCT R-IPR-18000198).

\section{Participants}

We plan to recruit 140 women with POR from participating hospitals via poster, networking, or WeChat. The posters will be placed on bulletin boards or other assigned places in the hospitals. In each participating hospital, at least one staff member (usually a postgraduate or doctor) will specialize in patient recruitment. Their contact information and clinic for screening visit will be detailed described in the recruitment advertisement.

Participants will be included if they meet the following criteria: (1) diagnosis with POR according to the Bologna criteria published in 2011 by the European Society of Human Reproduction and Embryology [1]; (2) age < 40 years; (3) previous POR ( $\leq 3$ oocytes retrieved in a conventional stimulation protocol); (4) an abnormal ovarian reserve test (i.e., AFC $<5-7$ follicles or AMH $<0.5-1.1$ $\mathrm{ng} / \mathrm{ml}$ ) or two episodes of POR after maximal stimulation; (5) suitability for GnRH antagonist protocol judged by physicians of reproductive medicine; and (6) voluntary participation and signing of the informed consent.

Participants will be excluded if they meet any of the following criteria: (1) history of ovarian surgery, radiotherapy or chemotherapy; (2) repeated miscarriages (including biochemical pregnancy) of more than 2 sessions; (3) repeated implantation failure (experienced at least 3 embryo transfer cycles, or failure to achieve clinical pregnancy after cumulatively transferring 10 good quality embryos); (4) uterine malformations (unicornate uterus, bicornuate uterus, uterus didelphys, or untreated uterus septus) or other untreated diseases that can damage the endometrial cavity such as adenomyosis, submucous myoma, intrauterine adhesions, and scarred uterus; 


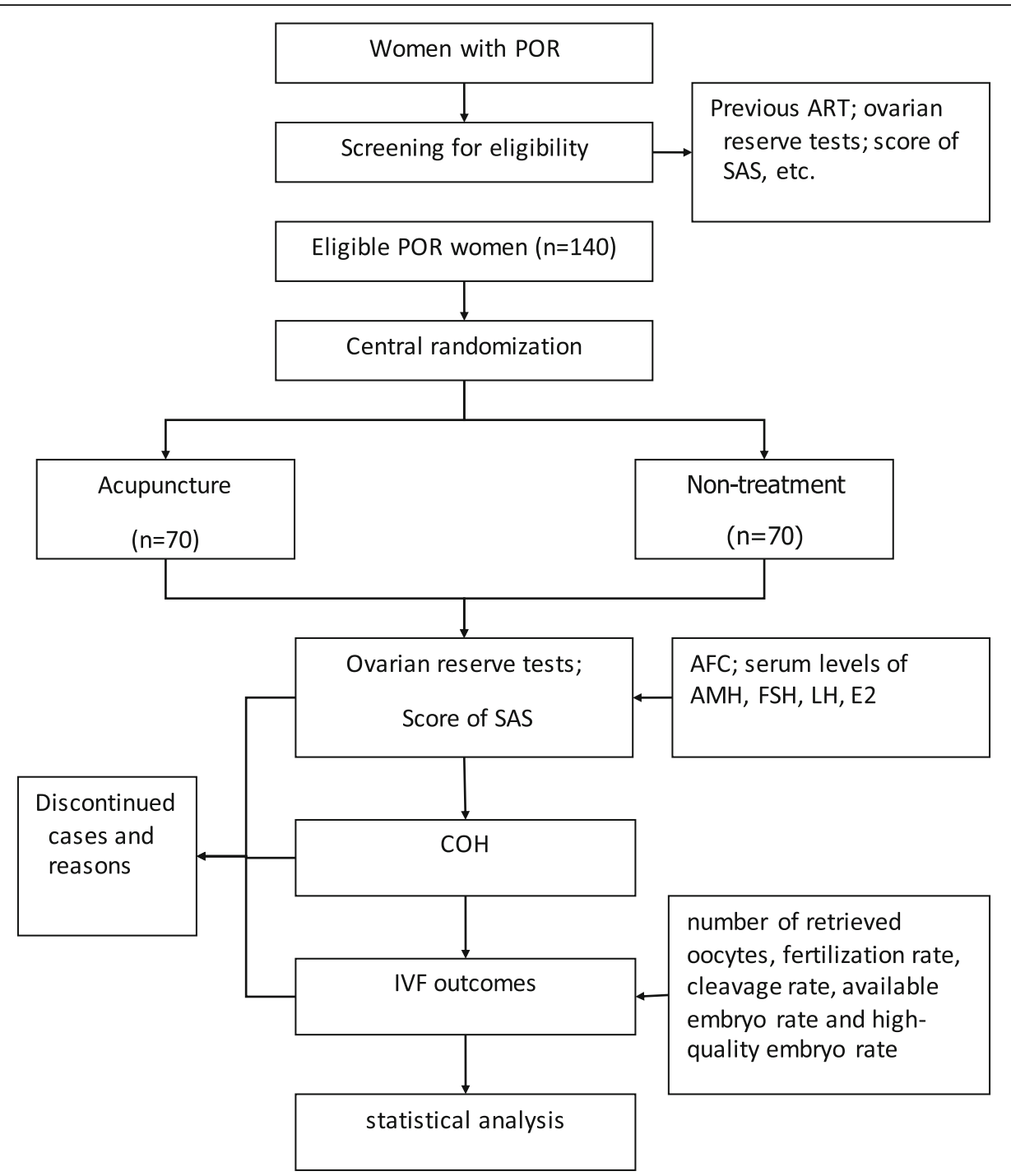

Fig. 1 Trial flow chart

(5) abnormal chromosomes in the patient or her partner (except chromosomal polymorphism); (6) untreated hydrosalpinx; (7) contraindications for pregnancy or ART; (8) untreated diseases that can negatively affect pregnancy such as high blood pressure, symptomatic heart disease, diabetes, liver or kidney disease, severe anemia, venous thrombosis, cerebral vascular diseases, and cancer; and (9) treatment with acupuncture or moxibustion treatment for POR in the previous 3 months.

\section{Randomization and masking}

Block randomization stratified by center will be applied in this trial. Eligible participants will be randomly assigned to acupuncture or nontreatment groups in a 1 : 1 ratio. The scheme of randomization including any planned restriction (e.g., blocking) will be designed and archived by a statistician who will not be involved in the statistical work of this trial. The allocation sequence will be computer-generated. If a participant is eligible for the trial, the appointed researcher (acupuncturist or reproductive physician) in each center will apply for the random number and group assignment via a telephone- and internet-based central randomization system of the Clinical Evaluation Center of the China Academy of Chinese Medical Sciences. A strict view permission will be set for the central randomization system, which allows no one except the top system administrator to view its randomization scheme. In this study, outcome assessors and data analysts will be blinded to group allocation.

\section{Interventions}

Acupuncture protocol

Patients in the acupuncture group will receive a 12-week acupuncture treatment regimen before $\mathrm{COH}$. Acupuncture 
Table 1 Study design schedule

\begin{tabular}{llll}
\hline Period & Screening & Baseline & Intervention \\
Week $(\boldsymbol{W})$ & $\times$ & W0 & W12 \\
\hline Informed consent & $\times$ & & \\
Eligibility & $\times$ & $\times$ & $\times$ \\
Demography and medical history & & $\times$ & $\times$ \\
AFC & & $\times$ & $\times$ \\
Serum level of AMH & & $\times$ & $\times$ \\
Basal serum levels of FSH, LH, and E2 & & $\times$ \\
Score of SAS & & $\times$ \\
Number of retrieved oocytes & & $\times$ \\
Number of fertilized oocytes & & $\times$ \\
Number of cleavage & & $\times$ \\
Number of available embryos & & $\times$ \\
Number of high-quality embryos & & $\times$ \\
AEs & & $\times$ & $\times$ \\
Compliance & & $\times$ & $\times$ \\
\hline
\end{tabular}

AFC antral follicle count, $A M H$ anti-Müllerian hormone, FSH follicle-stimulating hormone, $L H$ luteinizing hormone, E2 estradiol, SAS self-rating anxiety scale, AEs adverse events

will be performed by registered acupuncturists with over 2 years of experience. Hwato brand disposable acupuncture needles (size $0.25 \times 25 \mathrm{~mm}$, size $0.25 \times 40 \mathrm{~mm}$, and size $0.30 \times 75 \mathrm{~mm}$ ) and SDZ-III electroacupuncture apparatuses (Suzhou Medical Appliance Factory, Suzhou, China) will be used.

The acupuncture protocol designed for this trial is based on our clinical practice for improving ovarian function for women with DOR [14]. It consists of two groups of acupoints: Group 1 (in supine position): GV20 (Baihui), GV24 (Shenting), GB13 (Benshen), CV12 (Zhongwan), ST25 (Tianshu), CV4 (Guanyuan), KI12 (Dahe), EX-CA1 (Zigong), SP6 (Sanyinjiao), KI3 (Taixi), LR3 (Taichong); and group 2 (in prone position): BL23 (Shenshu) and BL33 (Zhongliao). These two groups of acupoints will be used alternatively with group 1 as the initial treatment. All acupoints will be located according to the World Health Organization Standardized Acupuncture Points Location [19]. A needle depth of 10 to $20 \mathrm{~mm}$ will be applied for GV20, GV24, and GB13 with transverse insertion and for LR3 with oblique insertion to the direction of KI1 (Yongquan). BL33 will be needled with an oblique downward trajectory of approximately 60 to $70 \mathrm{~mm}$ into the third posterior sacral foramina. The remaining acupoints will be perpendicularly needled for approximately 30 to $40 \mathrm{~mm}$. After insertion, small, equal manipulations of lifting, thrusting, or twirling will be performed on all needles (for GV20, GV24 and GB13, twirling only) to reach deqi, a composite of sensations (including soreness, numbness, distention, and heaviness) recognized as an essential component in achieving the acupuncture effect. In acupuncture with acupoints of group 2, paired electrodes from the electroacupuncture apparatus will be attached transversely to the needle handles at bilateral BL23 and BL33 with a dilatational wave and a current intensity that patients can tolerate (preferably with the skin around the acupoints shivering mildly without pain). Each acupuncture session will last for $20 \mathrm{~min}$. Participants will receive 3 sessions of acupuncture per week (ideally every other day) for 12 consecutive weeks for a total of 36 sessions.

\section{Nontreatment control}

Participants in the control group will receive nontreatment for 12 weeks before $\mathrm{COH}$.

\section{$\mathrm{COH}$ regimen}

Participant will start $\mathrm{COH}$ with a $\mathrm{GnRH}$ antagonist protocol on day 2 or 3 of the menstrual cycle after acupuncture or nontreatment period. GnRH antagonists are commonly used to prevent the premature LH surge that often occurs in poor responders [20,21]. This induces pituitary suppression within a few hours without a flareup effect, and the suppression is released immediately after their discontinuation [22]. Compared with $\mathrm{GnRH}$ agonist, GnRH antagonist protocol shows a shorter treatment time, a lower dosage of $\mathrm{Gn}$, and a similar clinical pregnancy rate [5]. In this trial, individualized GnRH antagonist protocol will be administered by physicians of reproductive medicine in each center. Given that participants may become suitable for $\mathrm{COH}$ before they complete the 12-week acupuncture or nontreatment, an early termination of acupuncture or nontreatment aiming to seize the opportunity to initiate a $\mathrm{COH}$ cycle will 
be allowed on the condition that they complete at least the 4-week acupuncture treatment (12 sessions of acupuncture) or nontreatment period.

\section{Permitted and prohibited concomitant treatments}

Participants will be discouraged from taking drugs that may interfere with the assessment of acupuncture effect, including Chinese herbs, Chinese patent medicine, sexual hormones, and contraceptives. If treatment not recommended in this trial has already been performed, relevant information should be recorded in patient's case report form.

\section{Outcome measures}

The primary outcome is the number of retrieved oocytes. The secondary outcomes include the assessment of ovarian reserve (AFC and serum levels of $\mathrm{AMH}, \mathrm{FSH}$, $\mathrm{LH}$, and E2), other IVF outcomes (fertilization rate, cleavage rate, available embryo rate, and high-quality embryo rate), and the score of the self-rating anxiety scale (SAS). Ovarian reserve will be assessed at baseline and after acupuncture or nontreatment period. AFC (via transvaginal ultrasound) and serum levels of $\mathrm{FSH}, \mathrm{LH}$, and E2 are required to be measured on days 2 to 5 of the menstrual cycle. AMH can be measured on any day of the menstrual cycle. SAS is a self-rating scale for measuring the presence and severity of anxiety [23]. A validated Chinese version of SAS will be used [24]. It consists of 20 items rated on a 4-point Likert scale. When using the scale, participants will be asked to rate each item from 1 to 4 points according to how it applies to them within the past week. The standard score is the sum of the integer part of 1.25 times the raw score (the sum of the 20 items ranging from 20 to 80 ). The presence of anxiety symptoms is defined as an SAS standard score of greater than 50. A higher score indicates a more serious case of anxiety. SAS will be assessed at baseline and after acupuncture or nontreatment period.

\section{Assessment of safety}

Adverse events (AEs) will be obtained by researchers by inquiring of the participants at each treatment or followup visit or by voluntarily reporting by the participants. Information of AEs will be appropriately documented throughout the trial. Acupuncture-related AEs include intolerable needling pain, bleeding after needle withdrawal, hematoma, and local infection. AEs related to $\mathrm{COH}$ include pain, organ injury, colporrhagia, and infection caused by egg retrieval, ovarian hyperstimulation syndrome, thrombosis, and allergic reaction.

\section{Data management and quality control}

A pretrial training will be done for all participating staff on the trial protocol, acupuncture manipulation, usage of the central randomization, and data management systems, etc. Double data entry will be applied in this trial to ensure the accuracy of data entry. A three-level quality control system (including self-inspection of each center, monitoring by project management group, and auditing by a third party) will be applied to ensure the implementation, recording, and reporting of clinical trials conform to the trial protocol, standard operation specifications, and relevant laws and regulations. Selfinspection will be done at least once every month by the quality controller of each center. On-site or remote monitoring will be done for all centers once every 3 months by the monitors appointed by the principle investigator. The auditing will be done by Clinical Evaluation Center of the China Academy of Chinese Medical Sciences at the beginning, middle, and end of the trial. An ethics committee will review conduct especially on safety, rights, and well-being of the participants at the middle and the end of the trial. Regular reminders via WeChat will be used to improve participant compliance. For patients who discontinue or deviate from the protocol, causes and relevant outcome data will be recorded in a case report form as much as possible.

\section{Statistical methods \\ Sample size calculation}

There are no available clinical studies on the effect of acupuncture for POR. Based on results of a TEAS study on POR [18], we assumed the mean (SD) number of retrieved oocytes would be 4.88 (1.84) in the acupuncture group and 3.95 (1.66) in the control group. A sample size of 57 participants per group would be needed to provide $80 \%$ power and a two-sided significance level of $5 \%$. Allowing for a $20 \%$ dropout rate, 140 participants will be recruited with 70 participants per group.

\section{Statistical analysis}

Statistical analysis will be performed by a statistician blinded to group assignments using SAS version 9.4 (SAS Institute Inc). All analyses will be based on the intentionto-treat principle. Missing data will be imputed using the multiple imputation method. Continuous data will be presented as the mean and standard deviation, or the median and interquartile range; categorical data will be presented as the number and percentage. Comparisons between groups will be analyzed using an independent $t$-test or Wilcoxon rank-sum test for continuous variables, and a chi-square test or Fisher exact test for categorical variables. All statistical tests will be two-sided, and $p<0.05$ will be considered statistically significant.

\section{Discussion}

This trial aims to determine whether acupuncture can improve follicular response to the $\mathrm{COH}$ during IVF 
cycles for women with POR. Several studies have shown that acupuncture improved ovarian reserve and reproductive outcomes in women undergoing IVF. However, the study population of these trials generally did not focus on women with POR. To date, there is only one study (a protocol published in 2018) [25] that aimed to assess the effect of acupuncture for POR. The present trial will be the second study using acupuncture treating POR. Compared with the published POR research [25], this trial will use the same control (nontreatment) and primary outcome (the number of retrieved oocytes), but a different acupuncture protocol and more secondary outcomes to better assess the acupuncture effect for POR. In this trial, nontreatment rather than placebo will be used as control due to the following reasons: placebo acupuncture is difficult to implement [26], and women are usually suggested to rest for 2-3 months between the two IVF cycles in clinical practice. It is worth mentioning that the acupuncture protocol of this trial is a mature protocol that has been used in our clinical practice for more than 8 years, and it has been shown to be effective in improving ovarian function [14, 15].

There are some limitations in this trial. We will only include women with POR aged under 40 who are suitable for $\mathrm{GnRH}$ antagonist protocol, which may weaken the representative nature of the sample. Based on clinical practice, the acupuncture protocol comprising two series of acupoints seems a little sophisticated for a randomized controlled trial, and it increases the difficulty in implementing placebo acupuncture. The results of this trial cannot expel the placebo effect, so it may exaggerate the effect of acupuncture by using nontreatment as control. This trial will not assess the effect of acupuncture on the end points of live birth rate or clinical pregnancy rate for POR women due to limitations of short observation period and too many confounding factors during IVF-ET.

In conclusion, the results of this trial will show the effect of acupuncture versus nontreatment in increasing number of retrieved oocytes and improving ovarian reserve for women with POR. This study will contribute to the research of acupuncture for POR worldwide by publishing results in a peer-reviewed journal.

\section{Trial status}

This trial is currently recruiting participants. The protocol version number and date: V1.0, May 9, 2018. The recruitment began on October 1, 2018. The estimated completion date of recruitment is February 29, 2021. The estimated study completion date is October 30, 2021.

\section{Abbreviations}

POR: Poor ovarian response; $\mathrm{COH}$ : Controlled ovarian hyperstimulation; IVF: In vitro fertilization; ART: Assisted reproductive technology;
GnRH: Gonadotrophin-releasing hormone; IVF-ET: In vitro fertilization-embryo transfer; DOR: Diminished ovarian reserve; POI: Premature ovarian insufficiency; FSH: Follicle-stimulating hormone; LH: Luteinizing hormone; AMH: Anti-Müllerian hormone; E2: Estradiol; AFC: Antral follicle count; TEAS: Transcutaneous electrical acupoint stimulation; RCT: Randomized controlled trial; SAS: Self-rating anxiety scale; AEs: Adverse events

\section{Acknowledgements}

Not applicable.

\section{Authors' contributions}

YGF and BYL conceived and designed the study protocol. HFX and CSZ drafted the manuscript. HFX completed the trial registration. HFX and CSZ and substantively revised the manuscript. LYH and HSY designed the statistical plan. TSS, HDW, YL, YB, GQT, LC, FZ, CZ, CLZ, YQY, MZH, and LY participated in data acquisition and provided administrative, technical, or material support. All authors have read and approved the submitted version of this manuscript and have agreed to be both personally accountable for their own contributions and to ensure that questions related to the accuracy or integrity of any part of the work.

\section{Funding}

This study was supported by the National key research and development program (NO. 2017YFC1703600) by the Ministry of Science and Technology of the People's Republic of China. The funder has no role in the design of the study and collection, analysis, and interpretation of data and in writing the manuscript.

\section{Availability of data and materials}

Not applicable.

\section{Ethics approval and consent to participate}

This trial will be performed in accordance with the Declaration of Helsinki and has been approved by ethics committees of all the participating centers. Written informed consent will be obtained from patients prior to enrolment. The name of the ethics committee and the reference number are as follows: Ethics committee (EC) of Institute of Acupuncture and Moxibustion, China Academy of Chinese Medical Sciences (2018-05-21-1);

EC of Shanxi Provincial Hospital of Chinese Medicine (AF/SC-04/01);

EC of Reproductive Hospital Affiliated to Shandong University (2018-05-21-1);

EC of Sun Yat-sen Memorial Hospital of the Sun Yat-sen University (2019-28);

EC of the first Hospital Affiliated to Lanzhou University (2018-05-21-1);

EC of reproductive medicine of Henan Provincial People's Hospital (SYZ-LL2019012411);

EC of Reproductive Medicine Center of Tongji Medical College Affiliated to Huazhong University of Science and Technology (2018-05);

EC of Shanghai Shuguang Hospital (2018-05-21-1);

EC of Nanjing General Hospital of Nanjing Military Command (2018-05-21-1);

EC of Luoyang Women and Children Health Care Center (2018-05-21-1).

\section{Consent for publication}

Not applicable.

\section{Competing interests}

The authors declare that they have no competing interests.

\section{Author details}

${ }^{1}$ Institute of Acupuncture and Moxibustion, China Academy of Chinese Medical Sciences, Beijing, China. ${ }^{2}$ Institute of Basic Research in Clinical Medicine, China Academy of Chinese Medical Sciences, Beijing, China.

${ }^{3}$ Shanxi Provincial Hospital of Chinese Medicine, Taiyuan, Shanxi, China. ${ }^{4}$ Shandong University Reproductive Hospital, Jinan, Shandong, China. ${ }^{5}$ Sun Yat-sen Memorial Hospital of the Sun Yat-sen University, Guangzhou, Guangdong, China. '́lanzhou University First Hospital, Lanzhou, Gansu, China. ${ }^{7}$ Henan Provincial People's Hospital, Zhengzhou, Henan, China. ${ }^{8}$ Huazhong University of Science and Technology Reproductive Medicine Center of Tongji Medical College, Wuhan, Hubei, China. ${ }^{9}$ Shanghai Shuguang Hospital, Shanghai, China. ${ }^{10}$ East Region Military Command General Hospital, Nanjing, Jiangsu, China. ${ }^{11}$ Luoyang Women and Children Health Care Center, Luoyang, Henan, China. 
Received: 29 September 2019 Accepted: 16 August 2020

Published online: 10 September 2020

\section{References}

1. Ferraretti AP, La Marca A, Fauser BC, Tarlatzis B, Nargund G, Gianaroli L, et al. ESHRE consensus on the definition of 'poor response' to ovarian stimulation for in vitro fertilization: the Bologna criteria. Hum Reprod. 2011;26(7):1616-24.

2. Polyzos NP, Devroey P. A systematic review of randomized trials for the treatment of poor ovarian responders: is there any light at the end of the tunnel? Fertil Steril. 2011;96(5):1058-61.e7.

3. Jaiswar SP, Natu SM, Sujata, Sankhwar PL, Manjari G. Prediction of poor ovarian response by biochemical and biophysical markers: a logistic regression model. J Obstet Gynaecol India. 2015:65(6):411-6.

4. Feldberg D, Farhi J, Ashkenazi J, Dicker D, Shalev J, Ben-Rafael Z. Minidose gonadotropin-releasing hormone agonist is the treatment of choice in poor responders with high follicle-stimulating hormone levels. Fertil Steril. 1994; 62(2):343-6.

5. Xiao J, Chang S, Chen S. The effectiveness of gonadotropin-releasing hormone antagonist in poor ovarian responders undergoing in vitro fertilization: a systematic review and meta-analysis. Fertil Steril. 2013;100(6): 1594-601.e1-9.

6. Sonmezer M, Ozmen B, Cil AP, Ozkavukcu S, Tasci T, Olmus H, et al. Dehydroepiandrosterone supplementation improves ovarian response and cycle outcome in poor responders. Reprod BioMed Online. 2009;19(4):508-13.

7. Chang EM, Han JE, Won HJ, Kim YS, Yoon TK, Lee WS. Effect of estrogen priming through luteal phase and stimulation phase in poor responders in in-vitro fertilization. J Assist Reprod Genet. 2012;29(3):225-30.

8. Fanchin R, Mendez Lozano DH, Schonauer LM, Cunha-Filho JS, Frydman R. Hormonal manipulations in the luteal phase to coordinate subsequent antral follicle growth during ovarian stimulation. Reprod BioMed Online. 2005;10(6):721-8.

9. Jeve YB, Bhandari HM. Effective treatment protocol for poor ovarian response: a systematic review and meta-analysis. J Hum Reprod Sci. 2016; 9(2):70-81.

10. Zhou L, Xia Y, Ma X, Tang L, Lu J, Tang Q, et al. Effects of "menstrual cyclebased acupuncture therapy" on IVF-ET in patients with decline in ovarian reserve. Zhongguo Zhen Jiu. 2016;36(1):25-8.

11. Manheimer E, Zhang G, Udoff L, Haramati A, Langenberg P, Berman BM, et al. Effects of acupuncture on rates of pregnancy and live birth among women undergoing in vitro fertilisation: systematic review and metaanalysis. BMJ. 2008:336(7643):545-9.

12. Xie ZY, Peng ZH, Yao B, Chen L, Mu YY, Cheng J, et al. The effects of acupuncture on pregnancy outcomes of in vitro fertilization: a systematic review and meta-analysis. BMC Complement Altern Med. 2019;19(1):131.

13. Isoyama D, Cordts EB, de Souza van Niewegen AM, de Almeida Pereira de Carvalho W, Matsumura ST, Barbosa CP. Effect of acupuncture on symptoms of anxiety in women undergoing in vitro fertilisation: a prospective randomised controlled study. Acupunct Med. 2012;30(2):85-8.

14. Li X, Xu H, Fang Y, Shang J, Yang H, Zhou X, et al. Acupuncture with regulating menstruation to promote pregnancy for diminished ovarian reverse: a prospective case series study (article in Chinese). China J Tradit Chin Med Pharm. 2017;37(10):1061-5.

15. Chen Y, Fang Y, Yang J, Wang F, Wang Y, Yang L. Effect of acupuncture on premature ovarian failure: a pilot study. Evid Based Complement Alternat Med. 2014;2014:718675.

16. Yang H, Fang Y, Xu H, Li X, Shang J, Yin Y. Systematic evaluation on the clinical efficacy of acupoint stimulation therapy for treatment of premature ovarian insufficiency on the basis of network meta-analysis. World $J$ Acupunct Moxibustion. 2017;27(3):26-39.

17. Jiao J, Jia T, Feng X, Sun W. Observation the effects and the changes of the TEAS in serum sex hormone sinus follicular number and $\mathrm{AMH}$ of the patients with ovarian poor response in 90 cases (article in Chinese). Chin J Birth Health Hered. 2017;8(25):114-6.

18. Lian F, Li R. Treatment of poor ovarian response with kidney deficiency by transcutaneous acupoint electrical stimulation combined intracavitary physiotherapy (article in Chinese). Chin J Integr Tradit West Med. 2017;37(5): 522-5.

19. Pacific WROftW. WHO standard acupuncture point locations in the Western Pacific region. Manila: World Health Organization; 2008.

20. Al-Inany $\mathrm{H}$, Aboulghar M. GnRH antagonist in assisted reproduction: Cochrane review. Hum Reprod. 2002;17(4):874-85.
21. Nikolettos N, Al-Hasani S, Felberbaum R, Demirel LC, Kupker W, Montzka P, et al. Gonadotropin-releasing hormone antagonist protocol: a novel method of ovarian stimulation in poor responders. Eur J Obstet Gynecol Reprod Biol. 2001;97(2):202-7.

22. $\mathrm{Pu} \mathrm{D}, \mathrm{Wu}$ J, Liu J. Comparisons of $\mathrm{GnRH}$ antagonist versus $\mathrm{GnRH}$ agonist protocol in poor ovarian responders undergoing IVF. Hum Reprod. 2011 26(10):2742-9.

23. Zung WW. A rating instrument for anxiety disorders. Psychosomatics. 1971; 12(6):371-9.

24. Liu L, Pang R, Sun W, Wu M, Qu P, Lu C, et al. Functional social support, psychological capital, and depressive and anxiety symptoms among people living with HIV/AIDS employed full-time. BMC Psychiatry. 2013;13:324.

25. Lee H, Choi TY, Shim EH, Choi J, Joo JK, Joo BS, et al. A randomized, open phase IV exploratory clinical trial to evaluate the efficacy and safety of acupuncture on the outcome of induction of ovulation in women with poor ovarian response: a study protocol for a randomized controlled trial. Medicine (Baltimore). 2018;97(34):e11813.

26. Chae $Y$. The dilemma of placebo needles in acupuncture research. Acupunct Med. 2017:35(5):382-3.

\section{Publisher's Note}

Springer Nature remains neutral with regard to jurisdictional claims in published maps and institutional affiliations.
Ready to submit your research? Choose BMC and benefit from:

- fast, convenient online submission

- thorough peer review by experienced researchers in your field

- rapid publication on acceptance

- support for research data, including large and complex data types

- gold Open Access which fosters wider collaboration and increased citations

- maximum visibility for your research: over $100 \mathrm{M}$ website views per year

At $\mathrm{BMC}$, research is always in progress.

Learn more biomedcentral.com/submissions 